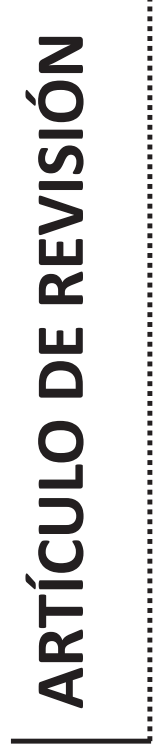




\title{
Lecciones de la participación ciudadana en el departamento del Caquetá frente al desarrollo de proyectos extractivos*
}

\section{Lessons from citizen participation in the department of Caquetá regarding the development of extractive projects}

Recibido: 18/05/2020 Aprobado: 17/07/2020

DOI: $10.25054 / 16576799.2724$

\section{RESUMEN}

Este artículo es resultado de una investigación realizada con ocasión de la Maestría en Derecho con énfasis en Derechos Humanos y Justicia Transicional de la Universidad del Rosario, e intenta presentar la situación de déficit de participación de las comunidades afectadas con el desarrollo de proyectos extractivos. Se pretende evidenciar que la realización de megaproyectos extractivos en el Departamento del Caquetá genera, de un lado, implicaciones sociales, económicas y culturales, y de otro lado, afecta los derechos humanos de la población involucrada. Por lo cual, se propone hacer una revisión del derecho y deber constitucional a la participación en Colombia, los mecanismos de participación y el desarrollo desigual que, frente a cuestiones socioambientales, ha tenido ese derecho respecto a diferentes poblaciones afectadas.

\section{PALABRAS CLAVE}

Mecanismos de Participación; Proyectos Extractivos; Derechos Humanos; Caquetá; Colombia.

\begin{abstract}
This article is the result of an investigation carried out on the occasion of the Master in Law with an emphasis on Human Rights and Transitional Justice at Universidad del Rosario, and attempts to present the situation of participation deficit of the affected communities with the development of extractive projects. This article aims to show that the carrying out of extractive megaprojects in the Department of Caquetá generates, on the one hand, social, economic and cultural implications, and on the other hand, affects the human rights of the population involved. Therefore, it is proposed to review the constitutional right and duty to participation in Colombia, as well as the participation mechanisms available to citizens to make their rights effective.
\end{abstract}

\section{KEYWORDS}

Participation Mechanisms; Extractive Projects; Human Rights; Caquetá; Colombia.

*Artículo de revisión. 


\section{INTRODUCCIÓN}

A través de este artículo se extraen las lecciones que el Estado colombiano debería asumir para evitar afectar el derecho a la participación de las comunidades campesinas asentadas en territorios en donde llegan a realizarse proyectos extractivos. Lo anterior es llevado a cabo haciendo una revisión documental y un estudio de casos, siendo ambas herramientas que hacen parte de la metodología cualitativa, que se caracteriza por enfocar su análisis partiendo del sujeto que experimenta y/o participa del fenómeno de estudio (Ruíz, 2012).

Como punto de partida se expondrá cómo se ha desarrollado el derecho a la participación, su concepto y alcance; seguidamente se hará un recuento de cómo se ha vivido este derecho en medio de los conflictos socioambientales. Posteriormente, se analizará el crecimiento de la actividad petrolera en el Departamento del Caquetá, Colombia. De ahí se expondrán dos casos en los que se ha evidenciado desconocimiento por parte del gobierno y de las multinacionales de la voluntad popular y cómo las comunidades han resistido a ello pacíficamente. Finalmente se invita a que, a partir de lo ocurrido, se tome conciencia de permitir a las comunidades afectadas una real participación, para lo cual será necesaria mayor presencia estatal, un dialogo sostenido entre el Estado y los ciudadanos y un mayor alcance de los mecanismos de participación y protección ciudadana.

Para hablar de participación es necesario reconocer su doble connotación de derecho y deber. En principio, la participación es una manifestación de la conducta humana, cambiante, que puede ser organizada o no, consciente o influenciada, y que por tanto es de diversos tipos y características. En líneas generales, la doctrina coincide en referirse a la participación como la posibilidad dada a las personas de tomar por sí mismas una cierta parte en el ejercicio del poder (Macías, 1998).
En Colombia, la noción de participación se relaciona con diversos derechos y principios consagrados en la Constitución Política de 1991, como la autonomía, el pluralismo jurídico, el principio democrático; y pese a la ambigüedad que puede tener dicho concepto a partir de diversas interpretaciones sobre su alcance, en los últimos años ha venido siendo reconocido como derecho fundamental por la jurisprudencia constitucional (CConst, T-129/2009, M. P. H. Sierra; CConst, T-348/2012, M. P. J. Pretelt) y ahora está consagrado legalmente como derecho en la Ley 1757 de 2015.

La participación es, al mismo tiempo, un derecho instrumental que permite lograr la defensa y reivindicación de otros diversos derechos, como el medio ambiente, el territorio, la seguridad alimentaria, la autodeterminación, entre otros (CConst, C-418/2002, M. P.A. Tafur).

De manera general y abstracta, puede decirse entonces, que la participación alude al derecho de tomar parte o iniciativa de las actividades que afectan el desarrollo y la proyección de la persona o a tener o tomar parte en las decisiones, asuntos 0 actividades públicas (Velásquez, 2003). Por ello, como lo expone Rodríguez (2014), la participación debe ser vista desde la perspectiva o enfoque de los derechos humanos, como marco conceptual que debe guiar los procesos de formulación, implementación y evaluación de planes y políticas públicas de desarrollo.

En este sentido, la participación ha tomado cardinal importancia para hacer frente a la violación 0 amenaza de derechos humanos de las diversas comunidades del Caquetá, en los lugares en que se adelantan proyectos extractivos, dado que en las últimas dos décadas la actividad de exploración y explotación de recursos naturales ha crecido exponencialmente impulsado por las políticas públicas adoptadas por los gobiernos de turno (Garcés y Rapalino, 2015). 


\section{PARTICIPACIÓN CIUDADANA FRENTE A CONFLICTOS SOCIOAMBIENTALES}

\subsection{Concepto y alcance del derecho a la participación}

Como lo precisan Rodríguez y Muñoz (2009), la participación no es un concepto estático sino de carácter dinámico que se manifiesta en diversas formas, espacios y niveles; puede ser utilizada de diferentes maneras y permite jugar en diversos escenarios y concretar intereses, lo que ha dado lugar a criterios legales y jurisprudenciales específicos. En este sentido, así como el concepto de participación tiene diferentes connotaciones, son también variados los tipos o formas de participación, que tienen algunos rasgos comunes, como, por ejemplo, que constituyen todos los ejercicios del derecho humano a participar, pero se diferencian en su alcance, contenido, efectos, actores involucrados, ámbito de aplicación etc. Entonces, sin la pretensión de abarcar en este trabajo todos los tipos o formas de participación, se intentará describir algunos, directamente relacionados con la cuestión que nos ocupa.

Una clasificación distingue los mecanismos de participación formal y no formal (Velásquez, 2003), para hacer referencia, con los primeros, a los establecidos y regulados por el ordenamiento jurídico político y, con los segundos, a cualquier otra forma de manifestación, organizada o no, no regulada expresamente en el ordenamiento jurídico pero adoptada planeada o espontáneamente para reivindicar o expresar alguna situación. Entre los primeros están los mecanismos creados por el Estado que, por ende, legitima su uso, como son el voto, el referendo, la consulta popular, el plebiscito, la iniciativa popular legislativa etc.; mientras que los segundos -los no formales o no regulados- hacen referencia, por ejemplo, a movilizaciones o marchas de ciudadanos, los llamados cacerolazos u otras formas de protestas colectivas (Hillón, 2014).

En concreto para el tema que nos ocupa en este artículo, hablaremos de la participación ambiental en el espacio territorial, puede tratarse de participación local o municipal, regional y nacional, dependiendo delárea de interés que involucra; siempre en relación con temas de incidencia en el respectivo espacio 0 área geográfica y de interés privado 0 estatal con incidencia en el medio ambiente y la gestión de los recursos naturales (Velásquez, 2003).

\subsection{Desarrollo del derecho a la participación frente a proyectos de desarrollo del alto impacto socioambiental}

En Colombia la consulta popular fue introducida al ordenamiento jurídico con el Acto Legislativo 01 de 1986, que en su artículo 6 establece que "(...) podrán realizarse consultas populares para decidir sobre asuntos que interesen a los habitantes del respectivo distrito municipal"; disposición que fue reglamentada mediante la Ley 42 de 1989. En ese entonces se limitó el objeto de las consultas a asuntos de orden local (municipal o distrital).

El 27 de agosto de 1995 se realizó la primera consulta popular en el municipio de Aguachica (Cesar) relacionada con la consecución de la paz; desde que entró en vigencia la Ley 134 de 1994, hasta junio de 2014 se habían realizado 33 consultas populares en el país sobre diversos temas (Garcés y Rapalino, 2015). Sin embargo, como forma de manifestación ciudadana frente a proyectos extractivos, el mecanismo ha ganado importancia desde 2013, con las consultas en los municipios de Piedras (Tolima) y Tauramena (Casanare); la primera, relacionada con el proyecto extractivo de La Colosa, y la segunda, mediante la cual se dijo "NO" la extracción petrolera en el territorio de ese municipio. En ese mismo año en el Municipio de Monterrey (Casanare) se intentó también realizar una consulta sobre un proyecto de extracción de hidrocarburos, pero fue suspendida por el Consejo de Estado.

En el año 2017 se realizaron siete consultas populares en los municipios de Cabrera (Cundinamarca) el 26 de febrero, Cajamarca (Tolima) el 26 de marzo, Cumaral (Meta) el 5 de junio, Pijao (Quindío) y Arbeláez (Cundinamarca) el 9 de 
julio, Jesús María (Santander) el 19 de septiembre, y Sucre (Santander) el 1 de octubre. Para 2018, según Anzola (2019), "ya había pendientes 54 consultas populares para frenar proyectos de minas y energía. En la mayoría de los casos, la Registraduría evitó que se realizaran aduciendo falta de presupuesto" (párr. 8).

En adelante hubo un estallido de consultas populares frente a proyectos extractivos, derivado en parte- de las experiencias anteriores en otros países latinoamericanos, su difusión en el contexto colombiano y el uso que los movimientos sociales empezaron a dar al referido marco político e institucional (Dietz, 2018). En todas las consultas en que se ha preguntado por el desarrollo de proyectos minero-energéticos ha sido mayor la votación en su contra.

Bajo la regulación de la Ley 134 de 1994, sólo el alcalde, gobernador o presidente podían promover consultas populares, pero la Ley 1757 de 2015 consagró la posibilidad de que se realizaran por iniciativa ciudadana, apoyada en la recolección de firmas, como fue la consulta anticorrupción realizada en el año 2018. En todo caso, para que la consulta se realice, independientemente de su origen (estatal o ciudadano), debe ser avalada por el Concejo Municipal, Asamblea Departamental o por el Congreso de la República; además, sigue teniendo como una de sus principales limitaciones la de recaer sobre asuntos atinentes a la circunscripción territorial dentro de la cual se convoque y desarrolle.

Aunque la consulta popular es un mecanismo legalmente regulado e incluso legitimado política y socialmente luego de su consagración constitucional, Dietz (2018) plantea que, ese solo hecho no garantiza los espacios políticos necesarios para su realización, la cual en algunos casos está determinada por cambios en las estructuras políticas e institucionales que influyen en las acciones de los promotores del mecanismo. Además de que, en Colombia, la regulación constitucional y legal de mecanismos, procedimientos y niveles de participación es prolija, pero en la práctica la rigurosidad de esta y la cantidad de requisitos impide que se materialice dicha participación y por el contrario terminan desestimulando a los ciudadanos (Velásquez, 2003).

Así, la consulta popular, mecanismo en el que los habitantes de los pueblos encontraron la forma de expresar su postura frente a planes y proyectos formulados y aprobados lejos de los territorios impactados, llegó a convertirse en instrumento de veto de proyectos extractivos $y$, por ende, en la piedra en el zapato de autoridades nacionales de Colombia (Dietz, 2018), cuyo modelo económico y presupuesto público -que han entrado a depender en buena medida de los ingresos derivados de la industria extractiva- se ven amenazados con el avance de manifestaciones ciudadanas contra ese tipo de proyectos.

Lo anterior ha conducido, de un lado, a que quienes apoyan las consultas se vean criminalizados, amenazados y estigmatizados por los partidarios de los proyectos extractivos, lo cual puede desestimular el importante proceso de movilización, previo a la votación de la consulta y, de otro lado, a que el gobierno -con la idea que de que el Estado es dueño del subsuelo, aunque los municipios sean los encargados de ordenar lo que existe encima de élintente limitar el alcance de este mecanismo en relación con tres dimensiones: temporal, espacial y técnica.

Ahora bien, los concejos de diferentes municipios empezaron a emitir acuerdos municipales restringiendo las actividades de extracción minera y de hidrocarburos en los territorios de su jurisdicción, pero los Tribunales Administrativos de los respectivos departamentos, como ocurrió en Antioquia y Huila, empezaron a declarar la invalidez de dichos actos por considerar que los concejos municipales no tenían competencia para decidir sobre la explotación del subsuelo. Así, el crecimiento exponencial de la industria extractiva en Colombia y las repetitivas manifestaciones ciudadana en su contra, a través de consultas populares, en los territorios impactados por esa actividad, ha puesto de 
relieve la tensión entre la autonomía de las entidades territoriales para decidir sobre su ordenamiento territorial y la facultad de la nación para decidir sobre políticas públicas de alcance nacional.

En concordancia con lo anterior, la Corte Constitucional, por medio de la Sentencia C-123 de 2014, dispuso que las actividades mineras no podían ser excluidas del territorio nacional sino por las autoridades competentes y declaró exequible el artículo 37 de la Ley 685 de 2001, en el entendido de que para autorizar actividades de exploración y explotación minera, las autoridades competentes del nivel nacional deben acordar con las autoridades territoriales concernidas, las medidas necesarias para la protección del ambiente sano, y en especial, de sus cuencas hídricas, el desarrollo económico, social, cultural de sus comunidades y la salubridad de la población, mediante la aplicación de los principios de coordinación, concurrencia y subsidiariedad.

De igual manera, en la Sentencia T-445 de 2016, la Corte Constitucional había expresado que la consulta popular está supeditada a las normas legales y constitucionales que la regulan y que su fuerza vinculante no puede desconocer otros derechos y principios reconocidos en la Constitución, por lo que no todo llamado a la comunidad para pronunciarse sobre asuntos de interés local puede concebirse en términos imperativos absolutos. Sin embargo, precisó que los entes territoriales poseen la competencia para regular el uso del suelo y garantizar la protección del medio ambiente, incluso si al ejercer dicha prerrogativa terminan prohibiendo la actividad minera.

La Corte Constitucional también se ha pronunciado en casos específicos, siendo un ejemplo de ello la Sentencia SU-095 de 2018. En esa oportunidad la Corte se apartó expresamente de la sentencia T-445 de 2016, por considerar que en ella se hizo una interpretación limitada y aislada de postulados y principios definidos en la Constitución Política, que la llevaron a autorizar a las autoridades locales para prohibir la minería. En este pronunciamiento, la
Corte estableció que ni la nación (nivel nacional o central) ni las entidades territoriales tienen competencias absolutas en materia de explotación del subsuelo y de los Recursos Naturales No Renovables (RNNR); entonces precisó que las entidades territoriales no tienen competencia absoluta ni poder de veto frente a actividades de explotación de los recursos del subsuelo.

Finalmente, las comunidades locales ya no podrán, directamente -mediante consulta popular- ni a través de sus representantes -miembros de los concejos municipales- impedir la ejecución de proyectos mineros o energéticos, pues mediante Sentencia C053 de 2019, la Corte Constitucional declaró inexequible el artículo 33 de la Ley 36 de 1994, que obligaba a realizar una consulta popular en los municipios donde un proyecto minero, turístico o de otro tipo amenazara con cambiar significativamente el uso del suelo. Con ello, la Corte continúa con la línea jurisprudencial iniciada con la Sentencia SU095 de 2018 en la que estableció que las consultas populares ya no son el mecanismo para prohibir actividades extractivas en los municipios.

La anterior decisión, beneficiosa para la inversión en proyectos extractivos de recursos naturales, privilegia un modelo de desarrollo guiado principalmente por criterios economicistas, sin un control efectivo por parte de las autoridades públicas y sin una posibilidad real de control social, puesto que la participación ciudadana quedó restringida, a través de mecanismos de democracia directa, y aun a través de organismos de representación. Persiste entonces la tensión entre la necesidad de una protección real del medio ambiente, cuyos daños se han ido tornando irreversibles, y la explotación de los recursos naturales para obtener recursos económicos para el país.

Ahora bien, aunque la Corte mantiene el postulado de que las entidades territoriales deben ser tenidas en cuenta por las autoridades nacionales para la autorización de actividades mineras y para concertar las medidas necesarias para la protección del medio ambiente y el desarrollo económico, social, cultural 
de sus comunidades y la salubridad de la población, mediante la aplicación de los principios de coordinación, concurrencia y subsidiariedad, esta no especifica, en la práctica, cómo debe hacerse dicha concertación, por lo que esa ambigüedad impedirá que se concrete una real y efectiva participación de las autoridades locales frente a los proyectos extractivos en sus territorios.

En este orden de ideas, estamos ante una exclusión de los municipios en asuntos relacionados con el ordenamiento minero que se desarrollen en su territorio, lo que se convierte en una mutilación de sus funciones, un atropello a la descentralización y un desconocimiento a la participación de la comunidad en las decisiones que los afectan, valor fundamental en un Estado de Derecho (Garcés y Rapalino, 2015, pp. 60-61).

Despojar de influencia a los entes territoriales y las comunidades que en ellos habitan de las decisiones que puedan afectar el ambiente y su forma de vida con el argumento del interés nacional y de que la propiedad del subsuelo es del Estado es una decisión contraria a un sistema democrático de un modelo de estado descentralizado y en el que el municipio está constitucionalmente consagrado como la entidad fundamental de la organización político-administrativa del Estado.

El interrogante es si esto servirá para construir un modelo de desarrollo menos conflictivo social y ambientalmente, pues no es viable adoptar un modelo en que unos municipios decidan permitir actividades extractivas y otros no; no solo porque la mayoría dirían que no y eso hace inviable una política pública nacional como la que debe tener por objeto este tipo de actividades, sino porque el Estado debe adecuar su modelo de producción a las manifestaciones ya hechas por la mayoría de la población, y aceptar que dichas actividades extractivas, al no estar reportando beneficios palpables y representativos para la población, no pueden pasar por encima del medio ambiente como derecho colectivo, ni vulnerar los derechos humanos de las poblaciones afectadas.
Lo anterior, teniendo en cuenta que el otorgamiento de licencias ambientales se hace desconociendo si es positivo o no el análisis económico costobeneficio, como herramienta -para determinar si el proyecto le conviene a la sociedad- que hace parte del estudio de impacto ambiental, que debe hacerse respecto de políticas públicas o proyectos, y que es necesario para decidir sobre una licencia ambiental solicitada (Vargas y Sarmiento, 2020).

\section{CRECIMIENTO DE LAACTIVIDAD PETROLERA EN EL CAQUETÁ Y LOS PROCESOS DE RESISTENCIAPOPULAR}

\subsection{Contexto minero en el Caquetá}

Desde los orígenes de la colonización del departamento del Caquetá, sus dinámicas se han caracterizado por el predominio de una economía extractivista, la concentración de tierras, las disputas de actores estatales y grupos armados por el control de la zona, además de la organización campesina como respuesta frente a la vulneración histórica de derechos (Ferro y Uribe, 2002). Productos como el caucho, la madera, los minerales y la coca han promovido la llegada y desplazamiento de habitantes tanto nacionales como provenientes de países vecinos a la zona, por lo que en el departamento la movilización continua y el desarraigo se han convertido en sucesos habituales.

Estas características son compartidas con otras zonas del sur del país, entre ellas Huila y Tolima, en las cuáles se encuentran tres de las fuentes de crecimiento económico más importantes para el país: los yacimientos de petróleo, la biodiversidad de la selva amazónica, oro y otros minerales y, áreas idóneas para la agricultura (Crudo Transparente, 2019). El país cuenta con 13 cuencas sedimentarias para exploración y extracción de hidrocarburos. De estas, dos quedan ubicadas en la región amazónica, la cuenca Caquetá-Putumayo y la cuenca Amazonas, la primera ampliamente explotada a diferencia de la segunda (Corpoamazonia, s.f.). Para efectos de esta investigación, se estudiará la cuenca Caquetá-Putumayo con énfasis 
en los efectos que ha tenido particularmente para el departamento del Caquetá y para sus pobladores.

Para el caso de la cuenca Caguán-Putumayo, desde el año 2002 se han adjudicado 26.699 hectáreas a la industria extractiva de crudo pesado, lo cual equivale al $42 \%$ del área habitable de todo el Caquetá que en total cuenta con 88.965 km2 (Díaz, 2015b). Para el año 2009 según Ciro et al. (2016) en la región existían "19 campos, 365 reservas de petróleo y 305 reservas de gas".

Actualmente, en el departamento del Caquetá existen 14 contratos o bloques petroleros y algunos se traslapan con resguardos 0 asentamientos indígenas como los Pijaos, La Libertad 2, Consara, Mecaya, Coropoya, el Libano, entre otros. Además, "las áreas que la ANH determinó como disponibles en la región, se encuentran ubicados 26 resguardos indígenas, de los departamentos de Putumayo, Guainía, Caquetá y Guaviare" (Prieto-Rozo, 2019, p. 7).

Ahora bien, la actividad petrolera que ya se realiza en el departamento está contaminando diferentes fuentes hídricas, como el Río Caguán, por el vertimiento de residuos tóxicos y por los derrames de petróleo dejan la tierra inservible y contamina el cuerpo de agua, obligando a las comunidades indígenas a abandonar poco a poco sus actividades tradicionales de siembra, pesca y caza. Este proceso de aculturación, que afrontan las comunidades étnicas, impide sus manifestaciones culturales, sus costumbres, sus conocimientos ancestrales, sus valores nacionales y culturales; dejando a la vista la irrefutable vulneración al derecho fundamental a la cultura y al territorio de las comunidades étnicas, la supervivencia física y cultural.

Para entender un poco el auge petrolero en la zona, es preciso entender que el departamento, como la gran mayoría del territorio nacional, ha contado con una problemática histórica frente a la acumulación de tierras por pequeños grupos de poder que se han aprovechado de las carencias institucionales, tales como, falta de intervención en la delimitación del territorio, ausencia de políticas migratorias y escaza o nula provisión de servicios públicos, para apropiarse de la tierra de manera violenta y masiva, mediante el despojo (Peña et al. 2014).

Estas ausencias estatales, han servido entre otros, para que grupos al margen de la ley encuentren en el departamento un lugar propicio para sus actos ilícitos (Centro Nacional de Memoria Histórica, 2013). Estas dinámicas han estado respaldadas por una arquitectura estatal débil tanto a nivel local como nacional (Díaz, 2015a), que ha dado lugar a que la propiedad de la tierra se concentre en narcotraficantes y clases terratenientes ganaderas (Centro Nacional de Memoria Histórica, 2013; González, 1989).

Bajo este contexto, la explotación de los recursos minero-energéticos apareció como una solución para generar desarrollo rural y fortalecer la economía (Díaz, 2015a), postura que con el paso de los años se ha estado fortaleciendo, especialmente desde la institucionalidad. Así, en su momento el Departamento Nacional de Planeación (2014) manifestó:

El sector minero-energético representa la oportunidad que tenemos de aprovechar de manera responsable nuestra riqueza de recursos naturales para generar crecimiento sostenible y mayor equidad social, regional e intergeneracional.

Las elevadas proyecciones de producción de petróleo y carbón para los próximos años, las estimaciones al alza de los precios internacionales de la canasta minero-energética y la creciente actividad de exploración en el territorio nacional muestran claramente el papel crucial que tendrá este sector en la economía colombiana en los próximos años (párr. 24).

Este auge extractivista, ha estado acompañado de negligencia estatal en los procesos de consulta previa y consulta popular, pues ni la Defensoría del pueblo, ni el Ministerio Público han ejercido control sobre estos mecanismos de participación, lo cual ha desembocado en violaciones a los derechos humanos de los pobladores, quienes a su vez en múltiples casos han sido también víctimas del 
conflicto armado, lo que genera revictimización, incremento en las tasas de subregistro, y desconocimiento de sus derechos y formas de protección(Díaz, 2015a).

Consecuentemente, el sector de hidrocarburos se ha posicionado de manera tal que, en muchas ocasiones los entes de control privilegian su actividad económica sobre los reclamos de protección de derechos de las comunidades (Negret, 2013), lo cual, entre otros, ha llevado a que las Zonas de Reserva Forestal sean utilizadas para la producción de crudo, ocasionando una devastadora oleada de despojos a comunidades campesinas e indígenas que incluso atenta contra su misma supervivencia. Lo anterior, bajo el argumento que señala que, como la tierra no está titulada, las comunidades no son propietarios de esta, sino solo de las mejoras que hayan hecho a los terrenos, razón por la cual las compañías extractivistas pueden poseerlas sin limitación alguna (Díaz, 2015a; Negret, 2013).

Frente a esto, la Corte Constitucional ha señalado que, en los procesos de autorización para la exploración y explotación minera, se deben tener en cuenta los aspectos de coordinación y concurrencia, basados en el principio constitucional de autonomía territorial. Para lo cual, las entidades locales deberán participar en los procesos "mediante acuerdos sobre la protección de cuencas hídricas y la salubridad de la población, así como, del desarrollo económico, social y cultural de sus comunidades" (CConst, C123/2014, M. P.A. Rojas).

Sin embargo, el gobierno y los entes de control en lugar de proteger y regular, han flexibilizado las condiciones para así facilitar, sin mayor rigor técnico ni jurídico, títulos mineros, licencias y permisos de exploración, olvidando por completo la planificación y el ordenamiento ambiental propio de cada territorio (Garay, 2013).

\subsection{Casos en los cuales se ha desconocido la voluntad popular en la extracción petrolera}

Caquetá continúa siendo hoy el escenario de movilizaciones sociales, las cuales son producto, entre otros, de la oposición de la comunidad a la realización de proyectos de exploración y explotación petrolera por parte de compañías de la industria (Crudo Transparente, 2019). La comunidad se opone principalmente a los impactos negativos en el medio ambiente que genera la industria hidrocarburífera, pues estos terminan teniendo repercusiones en la actividad agropecuaria en la que se basa su supervivencia y, por ende, termina impactando sus derechos fundamentales a una vida digna, a la cultura, a la seguridad alimentaria, entre otros.

Aunque la participación ciudadana hace parte de los pilares constitucionales respaldados por el gobierno en su discurso, en la práctica la apuesta económica del Estado en lo local propende hacia la extracción intensiva de recursos minerales, sobre todo si estos están concentrados en grandes cantidades en una zona determinada, como sucede en la Cuenca Caguán-Putumayo. Esta visión entra en confrontación con las aspiraciones de las comunidades, cuya apuesta es el desarrollo integral y armónico del territorio, teniendo como enfoque principal la promoción de actividades de tipo agrícola y ambiental, estando la actividad extractiva condicionada a la armonía con aquellas.

Hablar de voluntad popular en el Caquetá implica tener en el radar al $20 \%$ de la población, conformada por cinco comunidades indígenas (Sistema Nacional de Información Cultural, s.f.) y 143.569 campesinos que encuentran su fuente de sustento en el sector agropecuario principalmente (DANE, 2019). Estos pobladores dan cuenta de cómo los mecanismos de participación se han desnaturalizado para dar lugar a la formalización de proyectos que desconocen sus derechos y priorizan los intereses económicos de las élites. 
La Cuenca Caguán-Putumayo, que comparten los departamentos de Caquetá y Putumayo, concentrándose principalmente en territorio del primero, constituye una zona de alta importancia para el gobierno nacional, debido a que cuenta con un potencial de 6.000 barriles, en su mayoría de crudo pesado, que permitirían al país ser autosostenible energéticamente por un tiempo mayor al actualmente estimado, que es de 6.5 años, además de contar con reservas de gas y otros hidrocarburos (Crudo Transparente, 2019). Sumado a lo anterior, de acuerdo con la Agencia Nacional de Hidrocarburos (2016), más del 90\% del territorio del departamento está dispuesto para llevar a cabo actividades de extracción petrolera, sin embargo, en la actualidad solo el $25 \%$ es trabajado con este fin.

De allí que el gobierno nacional insista en promover el desarrollo de actividades de extracción petrolera en el departamento, lo que se ha potenciado a través de decisiones tales como el dejar de reconocer al departamento como parte de la Región Amazónica, para así dar concesión a empresas tanto nacionales como extranjeras extractivas, siendo el municipio de San Vicente del Caguán el que cuenta hoy en día con más contratos para la extracción de petróleo vigentes (Crudo Transparente, 2019).

Por su parte, los procesos de extracción petrolera en este municipio comenzaron como tal en el año 2006, durante la retoma del Caguán en uno de los intentos por lograr un proceso de paz con las FARC. Siendo este un proceso fallido, se presentó como una oportunidad para la empresa Emerald Energy, la cual empezó a hacer operaciones de sísmica en 2006 y en 2009 inició la explotación de crudo pesado (Barbosa y Ciro, 2016). A partir de ese momento, otras empresas extractivas - como Alange Energyintentaron incursionar en la zona, sin tener éxito debido al recrudecimiento del conflicto que se vivía en la zona.

Lo que han observado los pobladores del municipio es que, desde que Emerald Energy comenzó sus actividades, los precios de los alimentos y arriendos se incrementaron considerablemente, además de aumentar la dificultad para conseguir jornaleros en las fincas, pues los jóvenes ya no quieren dedicarse a actividades ganaderas 0 agrícolas (Crudo Transparente, 2019). Por otra parte, aunque los jóvenes estén estudiando carreras afines a la extracción petrolera, esto no garantiza su contratación en los proyectos locales y si logran trabajar, lo hacen desde la informalidad. Estas incursiones han generado en adición nuevas disputas entre el Estado y los grupos armados por el control del territorio, por lo que, aunque haya mayor presencia militar en la zona, esto no se ha traducido en mayor seguridad para los pobladores (Barbosa y Ciro, 2016).

Por estas razones, la comunidad se ha opuesto enfáticamente al desarrollo de proyectos petroleros en el departamento, no solamente en el municipio de San Vicente del Caguán, sino en otros espacios en los cuales, haciendo uso de vías de hecho y de mecanismos legales, han impedido o limitado la instalación de empresas en los territorios. Sumado a lo anterior, en febrero del año 2018, la comunidad presentó una iniciativa ciudadana cuyo objetivo consistió en posicionar nuevamente al Caquetá como un departamento Amazónico que propende por la defensa del agua, para volver así a ser objeto de la protección reconocida por el gobierno colombiano mediante la Ley 74 de 1979 en el Tratado de Cooperación Amazónica (Díaz, 2018).

Más recientemente, para el año 2016, el gobierno nacional concedió otra licencia de explotación en los pozos de San Vicente del Caguán a la empresa Emerald Energy, sumándose a otras veinte que ya han sido aprobadas (Crudo Transparente, 2019). Aunque estas actividades no llevan más de diez años realizándose en la zona, ya se han evidenciado efectos en el ecosistema derivados de la exploración y extracción de petróleo, además del incumplimiento de los parámetros establecidos desde el gobierno por las multinacionales adjudicadas para el desarrollo de dichas actividades.

Otro ejemplo de la desnaturalización de los mecanismos de participación en el departamento, es 
el proceso de consulta previa en la comunidad Inga, en el municipio de San José del Fragua, en el que le hicieron ofertas y atenciones al gobernador y a algunos líderes, y aunque el resguardo completo se encontraba en oposición, la empresa con el respaldo del liderazgo de la zona legalizó la consulta previa (Díaz, 2015a). Este tipo de actuaciones también ha generado afectaciones a las comunidades campesinas, quienes, a través de la compra de líderes sociales, o la utilización ilegal de sus firmas han visto formalizadas consultas populares (Díaz, 2015a; Posada y Sánchez, 2017).

En este sentido, los municipios de Curillo y Valparaíso viven la misma situación. A través de conductas intimidatorias y de soborno, las empresas extractivas han agilizado los procesos de consulta previa y los procesos de socialización, con la complicidad de un Estado ausente que no ejerce ninguna clase de control (Díaz, 2015a). Es evidente por tanto que "la manipulación de los procesos de consulta previa y socialización han dejado como saldo, el desplazamiento de varios campesinos, la contaminación de fuentes hídricas, la estigmatización de quienes se oponían al proyecto, y el soborno a líderes comunitarios" (Díaz, 2015b).

Concretamente en Valparaíso, aún con la renuencia de la comunidad, en el 2015, la empresa Emerald Energy construyó un pozo e inició el proceso de sísmica (Calle, 2018), gestionó su entrada contratando personas de la comunidad que debían visitar cada una de las fincas con la finalidad de obtener la aprobación individual de los campesinos, la cual respaldaron erróneamente mediante la firma con la que los campesinos dejaban constancia de que fueron visitados por la compañía, más no de su aceptación (Ciro et al. 2016).

Ante lo acontecido, la población campesina se vio muy vulnerada, pues no pudieron acceder a ningún otro mecanismo de protección, ya que, si bien hubieran querido llevar a cabo una consulta previa, esta solo aplica en la actualidad para comunidades étnicas. Los casos aquí detallados muestran cómo, a través de diferentes estrategias, incluso recurriendo al uso de la fuerza, estos proyectos son instalados en el territorio, pasando por encima de las peticiones y reclamos de la comunidad.

En el caso del campesinado se presenta un déficit de su derecho a la participación, ya que su protección constitucional es asimétrica respecto de otras comunidades (étnicas); por esto, luego de identificar una serie de particularidades de orden social, cultural y económico de la población campesina, que la hacen susceptible de maltrato y discriminación, Quesada (2013) propone que, a partir de reconocerlos como un sujeto culturalmente diferenciado, se amplíe a ellos el derecho a la consulta previa; frente a lo cual Hillón (2014), plantea que si bien la construcción de regímenes con diferentes niveles de participación según la movilización étnica que un grupo pueda realizar, convierte a los mecanismos de participación en espacios para excluir a aquellos que "se supone" que no poseen una identidad étnica, señala también que esa propuesta de ampliación del ámbito poblacional de la consulta previa no tiene en cuenta que ese instrumento no permite decidir sobre la puesta en marcha 0 no de un proyecto de desarrollo en un territorio, salvo cuando se aplica el consentimiento previo, libre e informado.

\subsection{Procesos de resistencia popular en el Caquetá}

Para el año 2013, de acuerdo con el Centro Nacional de Memoria Histórica (2017), en el Caquetá había en total 309 organizaciones registradas en la Oficina de Asuntos Comunitarios de la Secretaria de Gobierno, entre las cuales se encontraban asociaciones de productores, de profesionales, campesinas, de mujeres y de desplazados, entre otras que se han encargado de generar una articulación con la gobernación local y representar las exigencias de la comunidad frente a asuntos de carácter económico, social, político o ambiental.

No obstante, esta red de organizaciones no siempre ha logrado mantenerse consolidada, debido a la violencia que se ha dado en el territorio tras la llegada 
de diversos grupos armados a la zona, además de los conflictos persistentes con las multinacionales extractivas, las cuales llegan acompañadas por la Fuerza Pública, limitando así todo tipo de diálogo con las comunidades.

Frente a los casos revisados en el anterior apartado, se observa que, en los municipios de San José del Fragua y Valparaíso, por ejemplo, la violencia paramilitar tuvo un papel importante en la coerción de las comunidades campesinas, las cuales tuvieron que padecer de manera constante la guerra (Centro Nacional de Memoria Histórica, 2013). Bajo este escenario, su respuesta ha sido siempre pacífica, principalmente por el miedo que mantienen ante una posible ola de violencia.

Concretamente, frente a la llegada de la petrolera Emerald Energy y la socialización del proyecto, las comunidades campesinas, encabezadas por dirigentes campesinos como José Antonio Saldarriaga, empezaron a organizarse, a capacitarse y analizar las implicaciones que ocasionaría la incursión de la multinacional (Ciro et al. 2016). Como resultado, impulsados por lo acontecido en Valparaíso, cuatro de los cinco municipios afectados se opusieron a la actividad extractiva, especialmente por la amenaza al recurso hídrico, y de esta manera conformaron las Comisiones por la Vida del Agua. De esta red surgió la Mesa Departamental por la Defensa del Agua y del Territorio, que ha sido fundamental para articular los procesos de resistencia a nivel local (Ciro, 2015).

Por su parte, el municipio de San Vicente del Caguán es uno de los más representativos en materia de resistencia popular y movilización, siendo uno de los que, ante la incursión de la empresa Emerald Energy, preparó una Audiencia Pública Ambiental con el objetivo de "mostrar las irregularidades en los estudios de impacto ambiental y social presentados por la empresa china en el bloque el Nogal" (Díaz, 2018, párr. 15). Al igual que en Valparaíso, la resistencia de los campesinos en la zona ha sido llevada a cabo de manera pacífica, incluso cuando la respuesta de las multinacionales y de las Fuerzas Armadas sea de agresión ante las protestas.
Estos movimientos ciudadanos se han opuesto rotundamente a las actividades tanto extractivas como hidroeléctricas, pues consideran que su trabajo atenta contra el agua, los bosques y la biodiversidad en general (Sánchez, 2016). La defensa ambiental se ha realizado a través de la divulgación de información, espacios de formación y capacitación, así como manifestaciones de inconformismo, protestas y plantones, actividades en las cuales se exige específicamente que se respete el consentimiento previo, libre e informado de las comunidades campesinas e indígenas (Negret, 2013; Sánchez, 2016).

La fortaleza de las organizaciones comunitarias del Caquetá ha radicado en su capacidad para dialogar, negociar y llegar a acuerdos tanto con los actores armados como con funcionarios públicos, aún después de ser agredidos y desconocidos de ambas partes. Su resiliencia es la que les ha permitido dar pasos pequeños hacia el reconocimiento del Caquetá como un departamento que le apuesta a la sostenibilidad y lograr al mismo tiempo frenar el impulso extractivista que es promovido por el gobierno central, pasando incluso por encima de la legislación nacional y los tratados internacionales que han catalogado a la zona como lugar de protección ambiental especial, por hacer parte de la regiónAmazónica del continente.

\section{LECCIONESAPRENDIDAS}

Tras la promulgación del Plan de Desarrollo Nacional 2019-2022 "Pacto por Colombia, Pacto por la Equidad", en el cual se le dio al Ministerio de Minas y Energía el aval para conceder licencias de explotación en yacimientos no convencionales, la comunidad se ha opuesto con mayor énfasis al desarrollo de estas actividades, haciendo uso de diferentes mecanismos legales para promover una visión del territorio predominantemente agrícola.

Por ello, ante la situación de desacuerdo persistente entre las autoridades nacionales por un lado, y el gobierno departamental y la comunidad, por otro, frente al modelo económico y de desarrollo que se 
espera instaurar en el territorio, en este apartado se presentarán aquellas lecciones que se rescatan en el Caquetá, partiendo de la revisión de los casos de San Vicente del Caguán y Valparaíso, en relación con la participación de la comunidad, así como de los mecanismos a los cuales han acudido para buscar garantizar la defensa de sus derechos.

Primero, cabe rescatar la fortaleza en la organización comunitaria del Caquetá frente a la defensa del territorio, en la que se han involucrado pobladores, organizaciones de la sociedad civil, académicos y representantes de instituciones locales (Crudo Transparente, 2019). Lo que la comunidad exige por ende es que el Estado, que suele tomar decisiones desde Bogotá, les permita ser partícipes en estos procesos, teniendo en cuenta que son ellos quienes habitan y conocen el territorio, así como cada una de sus necesidades.

En este sentido el gobierno nacional y el gobierno local deben poner la lupa en el Caquetá. La comunidad debe ser reconocida por tanto como un actor clave en la toma de decisiones respecto de las dinámicas económicas y productivas del departamento, y deben ser tratados con un enfoque no solo territorial sino también diferencial, pues el departamento fue decretado como una zona priorizada para la implementación de los Acuerdo de Paz del gobierno con la guerrilla de las FARC, a través de los Programas de Desarrollo con Enfoque Territorial (PDET).

El Estado y la industria petrolera en vez de fortalecer la participación ciudadana en la zona, han optado por criminalizar a la comunidad que se ha manifestado en contra de los proyectos extractivos, pues como se vio en el apartado anterior, no han sido consultados ni involucrados en el proceso de toma de decisiones respecto a los asuntos que les concierne de manera directa; e incluso de manera fraudulenta han utilizado sus firmas para formalizar los procesos de socialización (Díaz, 2015a).

En consonancia con lo anterior, los procesos de toma de decisiones deben lograr conciliar la visión de desarrollo que tiene el Estado con la de las comunidades, procurando que toda nueva actividad que llegue al territorio tome como referente las perspectivas locales.

Segundo, la negativa del gobierno nacional hacia la generación de un diálogo con las autoridades locales y la comunidad evidencia no solo un desconocimiento de las dinámicas de la región, sino que también refleja un desinterés y falta de voluntad política por hacer que esta situación cambie. Sin esta voluntad, aunque se establezcan acuerdos con la comunidad y se propongan diferentes alternativas para conciliar las visiones de desarrollo, no se presentarán cambios progresivos.

Preocupa además el hecho de que, la Corte Constitucional haya limitado el desarrollo de consultas populares para definir asuntos territoriales relacionados con actividades extractivas, pues no especifica cuáles mecanismos alternos se podrían utilizar para generar un diálogo entre gobierno nacional, con las autoridades regionales y locales, en conjunto con las multinacionales y las comunidades, para llegar a un acuerdo. La limitación de la Consulta Popular y la falta de mecanismos legales para involucrarse en la concesión de licencias extractivas deja a la población aún más desprotegida frente a las medidas implementadas de manera arbitraria por el gobierno central en lo referente al uso del suelo local y la visión de desarrollo predominante.

Estas ausencias han generado profundos malestares, ya que, de un lado, las empresas han incumplido frecuentemente los procedimientos y aun así, las entidades encargadas les han expedido las licencias de exploración y explotación, lo que hace que lleguen a la zona con una actitud de distanciamiento y evasión frente a los reclamos y/o solicitudes presentadas por la comunidad, bajo el argumento de que sus actividades son avaladas por el gobierno central. De otro lado, las comunidades, tras años de vivir lo mismo, han acudido a las vías de hecho para manifestar públicamente su descontento e impedir la continuación de actividades. 
De allí la importancia de propiciar espacios de diálogo que vayan más allá de la contención temporal del conflicto, deben proponerse soluciones sostenidas y sustentables que satisfagan a las partes involucradas. Para lograr lo anterior, estos espacios deben centrarse en generar un debate sobre qué tipo de desarrollo se espera instaurar en el Caquetá, incluyendo la visión y necesidades de la comunidad, para lo cual es clave que los sistemas de participación se fortalezcan a nivel local, lo cual no se puede lograr sin mayor presencia estatal en el territorio.

En este mismo sentido se hace un llamado a volcar la mirada a las comunidades afectadas, permitirles la participación en la elaboración de políticas públicas proteccionistas de sus intereses y del territorio en especial. Cómo dice Ciro et al. (2016) no es suficiente escuchar a las comunidades, es necesario darles voz y voto en la planeación de sus territorios. Es menester entonces, que los ciudadanos puedan participar activamente en la toma de decisiones, que sus posturas frente a la conservación y cuidado de los recursos naturales sean tenidas en cuenta en las discusiones sobre la actividad extractiva.

Para lo anterior cobran especial relevancia los saberes, usos y costumbres ancestrales legados por los pueblos indígenas y tribales, que nos recuerdan que la naturaleza y el medio ambiente son un elemento de transversal importancia, por la necesidad de las actuales y futuras generaciones humanas de contar con un ambiente sano para llevar una vida digna y en condiciones de bienestar, pero sobre todo por la importancia de los demás organismos vivos con quienes se comparte el planeta, entendidas como existencias merecedoras de protección en sí mismas (CConst, T-622/2016).

Finalmente, se hace necesario desde la jurisprudencia ampliar los usos y alcances de los mecanismos de protección, pues, de un lado, la Corte Constitucional ha dicho que la consulta popular no puede impedir la actividad extractiva, porque, las decisiones sobre el uso del subsuelo deben ser tomadas por las autoridades nacionales en coordinación con las autoridades territoriales, no por las comunidades (CConst, SU-095/2018, M. P. C. Pardo).

De otro lado, si los afectados son campesinos no pueden ser sujetos de la consulta previa, pues es un mecanismo únicamente reconocido para comunidades étnicas. Ante esto, las comunidades se han quedado desprotegidas frente a proyectos que los afectan de forma significativa.

Por lo anterior, se invita a las altas cortes a reflexionar respecto de ampliar la cobertura de la consulta previa a las comunidades campesinas. En este sentido, la Corte Constitucional dio luces, pues reconoció como víctimas a los campesinos afectados por hidroeléctrica el Quimbo (CConst, T-135/2013, M. P. J. Palacio). De otro lado, le reconoció el derecho a la participación a comunidades campesinas afectadas por actividades mineras (CConst, SU-133/2017). Y, aunque ya se han tenido pequeños avances al respecto, hasta la fecha no se ha reconocido expresamente el derecho fundamental a la consulta previa para comunidades campesinas (Posada y Sánchez, 2017).

Y es que la misma Corte ha sostenido que, si se van a realizar actividades que impliquen el uso de los recursos naturales, los implicados deben procurar espacios de participación que den cuenta por parte de quienes vayan a verse afectados, si con esto se afecta la subsistencia de su grupo poblacional en particular. Para lograrlo es obligatorio realizar espacios de información y concertación (CConst, T348/2012, M. P. J. Pretelt; CConst, T-606/2015, M. P. J. Palacio).

Como se ha visto en el desarrollo de esta investigación esos espacios no se han dado. Y al ser limitado el uso de la consulta popular, solo queda disponible la consulta previa, la cual blindaría el derecho a la participación de las comunidades campesinas afectadas por la industria petrolera e incluso minera. Este mecanismo de protección se convertiría en el instrumento para preservar la integridad social, económica y cultural de las 
comunidades implicadas, pues es idóneo al permitir sopesar los intereses en conflicto para de determinar cuál de los dos es más legítimo.

\section{CONCLUSIONES}

Los casos de San Vicente del Caguán y Valparaíso son una muestra de los conflictos que persisten en el Departamento del Caquetá, derivados de las visiones encontradas frente al modelo económico y de desarrollo que se espera instaurar en el territorio, puesto que, a pesar de que en el discurso nacional el Estado ha manifestado tener la voluntad para incluir a las comunidades en aquellas decisiones que interfieren directamente en sus territorios, la realidad ha sido un tanto diferente.

Es claro que la voluntad de las organizaciones y de la comunidad para llegar a un acuerdo con las empresas y el gobierno es fuerte, siempre y cuando se respete su objetivo de preservar el ecosistema de la región y puedan robustecer las actividades ganaderas y agrícolas, por encima de la extracción de recursos. Lastimosamente, la voluntad de las multinacionales que tienen intereses en la zona y del gobierno nacional no se evidencia en sus acciones, sobre todo por el hecho de que, decisiones tales como el desconocer al Caquetá como parte de la región Amazónica y otorgar licencias a las empresas así estas no cumplan satisfactoriamente con los requisitos exigidos por la ley, demuestran que se ha buscado imponer estas actividades a cualquier precio.

Lo anterior ha llevado a las comunidades a recurrir a diversos mecanismos para hacer escuchar sus peticiones y exigir sus derechos, llegando incluso a hacer uso de vías de hecho. Esta situación se agrava si se tiene en cuenta que, cada vez se limitan más los mecanismos de participación a los cuales pueden acudir las organizaciones y los pobladores para involucrarse en la concesión de licencias a empresas extractivas.

La principal lección que busca dejar este artículo es por lo tanto la importancia de incluir las voces y perspectivas de la comunidad en los proyectos de extracción que se instauren en el departamento, más allá de simplemente recoger sus opiniones, sino que, como ya fue mencionado, permitirles involucrarse en las soluciones que se determinen por medio del diálogo. Quiénes mejor que los pobladores del Caquetá, para trabajar en los proyectos productivos y económicos del territorio, pues son quienes lo han habitado por años y conocen cuáles son los beneficios y perjuicios a los que se enfrentan.

Así mismo, la legislación debe convertirse en un aliado para promover la participación de las comunidades y la resolución de conflictos entre los actores que se involucran en el territorio, no debe ser utilizada por las autoridades para imponer su visión de desarrollo ni limitada por estas cuando los mecanismos logren promover la defensa y el respeto de los derechos fundamentales de los ciudadanos. Solo así se logrará conciliar el objetivo de generar ingresos para la nación y la visión de desarrollo sostenible que tienen las comunidades, ambas igual de importantes para el país.

\section{REFERENCIAS BIBLIOGRÁFICAS}

I. Anzola, M. (2019). Consultas populares: una muerte anunciada.

https://razonpublica.com/consultaspopulares-una-muerte-anunciada/

II. Agencia Nacional de Hidrocarburos (ANH). (2016). Mapa de Tierras, junio 13 de 2016, [en línea].

http://www.anh.gov.co/Asignacion-deareas/Paginas/Mapa-de-tierras.aspx

III. Barbosa, J. Y Ciro, A. (2016). Mapa petrolero de la Amazonia y resistencia en el Caquetá: retos de paz en el posconflicto. Bogotá: Centro de Investigación y Educación Popular. https://www.cinep.org.co/publicaciones/PD FS/20160801n.mapa_amazonia.pdf

IV. Calle, H. (2018). Los dudosos estudios ambientales de la explotación petrolera en Caquetá. Infoamazonía.

https://infoamazonia.org/es/2018/03/espan 
ol-los-dudosos-estudios-ambientales-dela-explotacion-petrolera-en-caqueta/\#!/ map $=51549$

V. Centro Nacional de Memoria Histórica. (2013). Caquetá: conflicto y memoria. Bogotá: CNMH.

http://www.centrodememoriahistorica.gov.c o/descargas/informes2014/cartillaCaqueta/ cartilla-caqueta-completa.pdf

VI. Centro Nacional de Memoria Histórica. (2017). La tierra no basta: Colonización, baldíos, conflicto y organizaciones sociales en el Caquetá. Bogotá: CNMH.

https://centrodememoriahistorica.gov.co/wp -content/uploads/2020/01/la-tierra-nobasta.pdf

VII. Ciro, E. (2015). Resistencia a la política extractivista en el Caquetá: De Valparaíso a El Doncello y Paujil. A la Orilla del Rio. http://alaorilladelrio.com/2016/05/03/resiste ncia-a-la-politica-extractivista-en-elcaqueta-de-valparaiso-a-doncello-y-paujil/

VIII. Ciro, E., Barbosa, J., y Ciro, A. (2016). En A. Ulloa, Y S. Coronado, Extractivismos y posconflicto en Colombia: retos para la paz territorial (págs. 413-440). Universidad Nacional de Colombia, Facultad de Ciencias Humanas.

https://www.cinep.org.co/publicaciones/PD FS/20160801.extractivismos_posconflicto_ completo.pdf

IX. Corpoamazonia, (s.f). Corporación para el Desarrollo Sostenible del Sur de la Amazonía.

http://www.corpoamazonia.gov.co/region/J ur_mineria.htm.

X. Crudo Transparente. (2019). La industria extractiva en la región Sur: De cara a las Próximas Elecciones Territoriales 2019. https://crudotransparente.com/2019/ 10/24/la-industria-extractiva-en-la-regionsur-de-cara-a-las-proximas-eleccionesterritoriales-2019/

XI. DANE. (2019). Censo general 2018. https://www.dane.gov.co/index.php/estadist icas-por-tema/demografia-y-poblacion /censo-nacional-de-poblacion-y-vivenda2018

XII. Departamento Nacional de Planeación. (2014). Bases del Plan Nacional de Desarrollo 2014-2018 "Todo por un nuevo país".

https://colaboracion.dnp.gov.co/CDT/Prens a/Bases $\% 20$ Plan $\% 20$ Nacional $\% 20 \mathrm{de} \% 20$ Desarrollo\%202014-2018.pdf

XIII. Díaz, K. (2015a). Incidencia de la política minero-energética sobre la tenencia de la tierra en el Caquetá(2012-2014).

Universidad del Rosario: Tesis de pregrado. https://repository.urosario.edu.co/bitstream/ handle/10336/11541/DiazParra-Karla Yubranny-2015. pdf? sequence $=4$ Yis Allowed=y

XIV. Díaz, K. (2015b). Petróleo y acumulación en el Caquetá: Una mirada al proyecto de expansión de la economía extractiva en la Amazonía colombiana. A la orilla del río. http://alaorilladelrio.com/2015/02/17/petrole o-y-acumulacion-en-el-caqueta-unamirada-al-proyecto-de-expansion-de-laeconomia-extractiva-en-la-amazoniacolombiana/

XV. Díaz, K. (2018). ¿Qué implica que el Caquetá vuelva a ser integralmente amazónico y protector del agua? Revista Semana Sostenible.

https://sostenibilidad.semana.com/opinion/ articulo/iniciativa-ciudadana-del-caquetapara-oponerse-a-la-explotacionpetrolera/39681

XVI. Dietz, K. (2018). Consultas populares mineras en Colombia: Condiciones de su realización y significados políticos. El caso de La Colosa. Colombia Internacional, (93), 93-117.

https://revistas.uniandes.edu.co/doi/pdf/10. 7440/colombiaint93.2018.04

XVII. Ferro, J. y Uribe, G. (2002). Las marchas de los cocaleros del departamento de Caquetá, Colombia: contradicciones políticas y obstáculos a la emancipación social. Cuadernos de Desarrollo Rural, (49), 59-84. 
http://www.mamacoca.org/docs_de_base/ Cifras_cuadro_mamacoca/ferroMedina_m archascocaleras.pdf

XVIII. Garay, L. (2013).

Globalización/glocalización, soberanía y gobernanza. A propósito del cambio climático y el extractivismo minero. En L. Garay, Minería en Colombia (págs. 9-19). Contraloría General de la República.

https://www.contraloria.gov.co/documents/2 0181/472306/01_CGR_mineria_I_2013_co mp.pdf/40d982e6-ceb7-4b2e-8cf25d46b5390dad

XIX. Garcés, M. Y Rapalino, W. (2015). La Consulta Popular como mecanismo de participación ciudadana para evitar actividades mineras. Justicia Juris, 11(1), 52-62.

http://www.scielo.org.co/pdf/jusju/v11n1/v1 1n1a05.pdf

XX. González, J. (1989). La Colonización Marginal y las nuevas fronteras colombianas. En Análisis. Conflicto social y violencia en Colombia. Documentos ocasionales. CINEP

http://bibliotecavirtual.clacso.org.ar/Colomb ia/cinep/20100920125424/art2Controversi a189.pdf

XXI. Hillón, Y. (2014). La participación en conflictos socioambientales: la paradoja institucional colombiana. En Eslava, Adolfo (Ed.) Oro como fortuna. Instituciones, capital social y gobernanza de la minería aurífera colombiana (pp. 410-449). Medellín, Colombia: Universidad EAFIT.

https://www.eafit.edu.co/centros/analisispolitico/Documents/2.\%20Libro\%200ro\%2 0como\%20fortuna.pdf

XXII. Macías, L. (1998). Introducción al Derecho Ambiental. Bogotá: Legis.

XXIII. Negret, R. (2013). Derechos, Minería y conflictos. Aspecto Normativo. En L. Garay, Minería en Colombia. Fundamentos para superar el modelo extractivista (págs. 2354). Contraloría General de la República. https://redjusticiaambientalcolombia.files.w ordpress.com/2013/05/mineria-encolombia-fundamentos-para-superar-elmodelo-extractivista2013.pdf

XXIV. Peña, R., Parada, M., Y Zuleta, S. (2014). La Regulación agraria en Colombia o el eterno déjavu hacia la concentración y el despojo: un análisis de las formas jurídicas colombianas sobre el agro (1991-2010). Estudios Socio-jurídicos, 16, 121-164. https://revistas.urosario.edu.co/index.php/s ociojuridicos/article/view/esj16.1.2014.03/2 379

XXV. Posada, C., y Sánchez, D. (2017). Derecho a la consulta previa para las comunidades campesinas. Trabajo de grado: Universidad Eafit.

https://repository.eafit.edu.co/bitstream/han dle/10784/12390/Carolina_PosadaMesa_D aniela_S\%C3\%A1nchezYepes_2017.pdf?s equence $=2$ YisAllowed=y

XXVI. Prieto-Rozo, Andrea, (2019), Petróleo en la Amazonia ¿Pueblos indígenas en peligro?, Asociación Ambiente y Sociedad. https://www.ambienteysociedad.org.co/Des cargar/4612/banca_megaproyectos_/2364 8/boletin_hidrocarburos_amazonia_comuni dades_indigenas_compressed.pdf

XXVII. Quesada, C. (2013). Derecho a la Consulta Previa para comunidades campesinas. Universidad Nacional de Colombia, 1-119. http://www.bdigital.unal.edu.co/9859/1/701 683.2013.pdf

XXVIII. Rodríguez, G. Y Muñoz, L. (2009). La participación en la gestión ambiental. Un reto para el nuevo milenio. Bogotá, Colombia: Universidad del Rosario.

XXIX. Rodríguez, G. Y Gómez, A. (2013). La Participación como mecanismo de consenso para la asignación de nuevos derechos. Revista Pensamiento Jurídico, (37), 71-104. https://revistas.unal.edu.co/index.php/peju/ article/view/41191

XXX. Rodríguez, G. (2014). De la consulta previa al consentimiento previo libre e informado a pueblos indígenas en Colombia. Bogotá, Colombia: Universidad del Rosario. 
XXXI. Ruíz, J. (2012). Metodología de la investigación cualitativa. Bilbao: Universidad de Deusto.

XXXII. Sánchez, F. (24 de noviembre de 2016). Caquetá: el temor ante la minería y las hidroeléctricas en la Amazonía colombiana. https://es.mongabay.com/2016/11/caquetatemor-ante-la-mineria-las-hidroelectricasla-amazonia-colombiana/

XXXIII. Sistema Nacional de Información Cultural. (s.f.). Caquetá.

http://www.sinic.gov.co/SINIC/ColombiaCul tural/ColCulturalBusca.aspx?AREID=3YSE CID=8YldDep=18YCOLTEM=216\#: :text= En\%20Caquet\%C3\%A1\%20actualmente\% 20viven $\% 20$ cinco,municipios $\% 20$ de $\% 20 \mathrm{Mi}$ $1 \%$ C3\%A1n\%20y\%20Solano.

XXXIV. Velásquez, C. (2003). Participación Ambiental, Mecanismos establecidos por los Estados Español y Colombiano Para la defensa del medio ambiente y los recursos naturales. Revista De Derecho, Universidad Del Norte, (20), 135-197.

https://www.redalyc.org/pdf/851/85102007. pdf

XXXV. Vargas, A. y Sarmiento, J., Díaz, D., (2020). Has Cost Benefit Analysis Improved Decisions in Colombia? Evidence from the Environmental Licensing Process. E cological Economics, https://doi.org/10.1016/j.ecolecon.2020.10 6807

\section{REFERENCIAS JURISPRUDENCIALES}

XXXVI. CConst, C-180/1994, M. P. H. Herrera. XXXVII. CConst, C-418/2002, M. P. A. Tafur. XXXVIII. CConst, T-129/2009, M. P. H. Sierra. XXXIX. CConst, T-763/2012, M. P. J. Pretelt. XL. CConst, T-348/2012, M. P. J. Pretelt.

XLI. CConst, T-135/2013, M. P. J. Palacio.

XLII. CConst, T-172/2013, M. P.J. Palacio.

XLIII. CConst, C-123/2014, M. P.A. Rojas.

XLIV. CConst, T-294/2014, M. P. M. Calle.

XLV. CConst, C-623/215, M. P. A. Rojas.

XLVI. CConst, T-606/2015, M. P. J. Palacio.
XLVII. CConst, T-766/2015, M. P. G. Mendoza.

XLVIII. CConst, C-035/2016, M.P. G. Ortiz.

XLIX. CConst, T-445/2016, M. P. J. Palacio.

L. $\quad$ CConst, T-704/2016, M. P. L. Vargas.

LI. CConst, SU-095/2018, M. P. C. Pardo.

LII. CConst, C-053/2019, M. P. C. Pardo.

\section{REFERENCIAS COMPLEMENTARIAS}

LIII. Agencia Nacional de Hidrocarburos. (s.f). Estudios integrados del CaguánPutumayo.

http://www.anh.gov.co/InformacionGeologica-y-Geofisica/EstudiosIntegrados-y-Modelamientos/Presenta ciones $\% 20$ \% 20 Poster $\% 20$ Tcnicos/Tipo\% 201\%20(PDF).pdf

LIV. Asamblea General de las Naciones Unidas. (1948). Declaración Universal de los Derechos Humanos.

https://www.ohchr.org/EN/UDHR/Documen ts/UDHR_Translations/spn.pdf

LV. $\quad$ Fundación Paz y Reconciliación. (2014). Candidatos y nexos con fuerzas ilegales. Informe elecciones legislativas 2014. https://www.elpais.com.co/elpais/archivos/ candidatos-con-presuntos-nexosilegales.pdf

LVI. Reveles, F. (2017). Democracia participativa para el fortalecimiento de la representación política. La experiencia latinoamericana. Andamios, 14(35), 71-97.

http://www.scielo.org.mx/scielo.php?script =sci_abstractYpid=S1870-0063201700 0300071Ylng=esYnrm=iso

LVII. Zovatto, D. (2007). Las instituciones de la democracia directa a nivel nacional en américa latina: Un balance comparado: 1978 - 2007. Revista de Derecho Electoral,(4).

https://www.researchgate.net/publication/2 69970784_Las_instituciones_de_la_demo cracia_directa_a_nivel_nacional_en_Amer ica_Latina_Balance_comparado_19782010. 